\title{
Editorial
}

\section{The triumph of antibiotics and bacterial resistance}

The historical scourge known as the bubonic plague killed up to one-third of Europe's population in the 1300s. Infectious bacteria that cause pneumonia, ear infections, acne, gonorrhea, urinary tract infections, meningitis, and tuberculosis, can now outwit some of the most commonly used antibiotics and their synthetic counterparts, antimicrobials. For all these reasons, antibiotic resistance has been a problem for nearly as long as we've been using antibiotics. Not long after the introduction of penicillin in the 40s of last century, a bacterium known as Staphylococcus aureus began developing penicillin-resistant strains. More and more hospital-acquired infections are resistant to the most powerful antibiotics available, methicillin and vancomycin. These drugs are reserved to treat only the most intractable infections in order to slow development of resistance to them. So antibiotic resistance is not a new problem, medical community accepts penicillin and other antibiotic as miracle drugs for their ability to cure serious and often life threatening infections that are challenged by some defiant strains of bacteria.

A key factor in the development of antibiotic resistance is the ability of infectious organisms to adapt quickly to new environmental conditions. Bacteria are single-celled creatures that, compared with higher life forms, have small numbers of genes. Therefore, even a single random gene mutation can greatly affect their ability to cause disease. And because most microbes reproduce by dividing every few hours, bacteria can evolve rapidly. A mutation that helps a microbe survive exposure to an antibiotic drug will quickly become dominant throughout the microbial population. Microbes also often acquire genes, including those that code for resistance, from each other.

The triumph of antibiotics over disease-causing bacteria is one of modern medicine's greatest success stories. But many antibiotics do not pack the same punch they once did with their repeated use. Today whispered use of antibacterial in animal feed; consumer products like toiletries provide a great opportunity for the development of bacterial resistance. We are dealing with the living microbes that have shown incredible ability to accommodate and come out wining. Prescribers are sometimes quick to prescribe antibiotics for even flu and common cold.

Even when used carefully, all organisms can develop some resistance to antibiotic over time. It is perfectly natural phenomenon for a living organism to develop the means of survival in the hostile environment. Patient can do their part to help curb resistance by not demanding the unnecessary antibiotic and by finishing the prescribed duration even if the symptoms disappeared.

Frequent and thorough hand washing is key tom prevent the spread of infection. Good kitchen habits such as storing foods in proper temperature, washing fruits and vegetables thoroughly and cooking foods completely can also reduce the chance of getting food borne illness. Many medical physicians admit that patients expect and even demand antibiotic usage on their children with these types of problems. Doctors have lost patients from their practice when they have refused to administer antibiotics to a parent who mistakenly believes that form of care is needed. Nevertheless, up to $60 \%$ of children with common colds are treated 
with antibiotics (Journal of Family Practice 1996; 42:357--361). Because children average three to eight colds each year, most accompanied by green or yellow runny noses, they can get many, many rounds of unnecessary (and therefore harmful) antibiotics.

Scientists fear that certain bacteria that develop resistance in animal s can then infect people who eat meat or animal products. Tetracycline, fluoroquinolones are widely used today for farm animals. The risk of use of antibiotics in animal is difficult to measure precisely the impact on human health. Expert believes that already resistant strains of salmonella, campylobacter, enterococcus and E.coli have been transmitted from animals to human. As long as antimicrobial drugs are used, drug resistance will remain a challenge. But the educational and behavioral interventions for modifying drug prescribing practice for health care providers and patients on appropriate use of antibiotics and adherence to prescription instructions are only the ways to reduce the emergence of bacterial resistance against antibacterials. 